Voix et Images

voixetimages

\title{
Paradigme, palimpseste, pastiche, parodie dans Maryse de Francine Noël
}

\section{Anne Élaine Cliche}

Volume 12, numéro 3 (36), printemps 1987

Yves Beauchemin

URI : https://id.erudit.org/iderudit/200657ar

DOI : https://doi.org/10.7202/200657ar

Aller au sommaire du numéro

Éditeur(s)

Université du Québec à Montréal

ISSN

0318-9201 (imprimé)

1705-933X (numérique)

Découvrir la revue

\section{Citer cet article}

Cliche, A. É. (1987). Paradigme, palimpseste, pastiche, parodie dans Maryse de Francine Noël. Voix et Images, 12(3), 430-438. https://doi.org/10.7202/200657ar d'utilisation que vous pouvez consulter en ligne.

https://apropos.erudit.org/fr/usagers/politique-dutilisation/ 


\title{
Paradigme, palimpseste, pastiche, parodie dans Maryse de Francine Noël
}

\author{
par Anne Élaine Cliche, Université d'Ottawa
}

(...) l'attirance qu'elle avait toujours éprouvée pour le personnage d'Elisa Doolittle s'expliquait parfaitement. Elle se dit: "Oui, c'est $c$ a, c'est un trip de langage! J'ai toujours fait des trips de langage et, au couvent, c'est comme ça que je m'en suis sortie.

Maryse $^{1}$, p. 365

- Il paraît qu'elle a commencé à écrire.

- Tiens, une autre! On voudrait toutes écrire, finalement. Qu'est-ce qu'on a? Qu'est-ce qu'on veut prouver? Qu'est-ce qui nous manque?

Maryse, p. 156

Ecrire. Préoccupation qui pose toujours d'avance la question du lieu. D'où écrire? ou plutôt d'où écrit-on? Question fondamentale puisqu'elle met en lumière l'inscription d'une conscience, de toute conscience, à l'intérieur d'une culture et du langage (des langages) qui la produit.

Depuis le début du siècle, les recherches de Bakhtine, Todorov, Kristeva, Grivel ou Barthes ont montré à quel point tout texte s'articule sur l'infini des textes et des discours qui l'entourent et le précèdent. Le dialogisme de Bakhtine $^{2}$ et la notion d'intertextualité redéfinie par Kristeva ne viennent que souligner et mettre à jour la situation de l'œuvre littéraire qui, aujourd'hui, se produit et se définit dans une réalité reconnue comme langage.

On remarque par ailleurs qu'il est devenu usuel (depuis le structuralisme et surtout depuis Lacan) de faire du «texte» une notion très élargie. Lorsque Julia Kristeva définit le texte en système de signes ${ }^{3}$, il ne s'agit plus simplement de renvoyer à une ouvre littéraire ou à un écrit mais de rejoindre les différents types de discours en circulation dans une société, voire les systèmes symboliques eux-mêmes, sociaux ou inconscients.

Si écrire est toujours réécrire, on doit saisir dans cette "réécriture» un processus de déplacement, de transposition, dans lequel les textes (intertextes) ne peuvent plus assurer une réelle autonomie, et oủ les codes ne parlent plus d'un seul lieu mais sont parlés par un ensemble qui les prend en charge.

(...) tout texte se construit comme mosaique de citations, tout texte est absorption et transformation d'un autre texte, avec tous les textes et avec l'histoire elle-même considérée comme texte ${ }^{4}$.

L'intertextualité apparaît donc à la fois comme polyvalence implicite, décentrement idéologique, écriture multidirectionnelle et moyen de subversion. Ce dernier aspect renvoie à des formes bien particulières du dialogisme: le pastiche et la parodie. 
On connaît bien les travaux de Linda Hutcheon ${ }^{5}$ sur cette question. Dans ces réflexions théoriques elle reconnaît dans la parodie une stratégie "métafictionnelle» propre à organiser les textes sur un mode de distanciation et de différence critique:

En réalité, c'est la stratégie consistant à faire jouer ensemble des différences littéraires, culturelles, et même morales, telle qu'elle est impliquée par le contraste parodique formel, qui contribue le plus à assurer une place privilégiée à la parodie dans le roman ironique moderne 6 .

Cette stratégie se développerait, selon Hutcheon, en marge du pastiche qui se présenterait alors comme une forme essentiellement monotextuelle. Pour les fins de cette analyse, nous considérerons au contraire le pastiche dans son acception la plus générale, c'est-à-dire comme redoublement amplifié, différé, non pas dans une pure intention imitative mais dans une double articulation de référence ( «trace» du texte pastiché) et de distanciation (excès, débordement), le posant ainsi dans une fonction parodique formelle.

Le pastiche est en fait le lieu de jouissance du rapport entre deux textes. Il est aussi le dire d'une distance critique, la conscience ludique du caractère double (au moins) de tout écrit, l'excès du texte manquant.

Cette conscience du constant dialogue qu'entretiennent les textes entre eux entraîne le refus de revendiquer une soi-disant "inviolabilité" littéraire et rend compte d'une autre prise de conscience marquant la littérature contemporaine: celle du décentrement du sujet lié à une explosion des écritures et des lectures du sens mais aussi celle d'une "métafiction" généralisée à partir d'une impossible adéquation au réel et au langage.

Le pastiche, le jeu de mots, les anagrammes manifestent une attitude qui privilégie le langage dans sa matérialité même. Que de nombreuses femmes écrivains se soient intéressées à une telle pratique de l'écriture intertextuelle 7 avec sa part de ludique vient peut-être signifier, pour elles, la reconnaissance d'un lieu propice où le langage n'a plus fonction d'affirmer le pouvoir d'une raison univoque et propriétaire, mais où peut se dire une différence qui soit d'abord conscience d'une circulation infinie des discours, des voix et des pouvoirs à travers nous.

Ainsi, les écritures de Nicole Brossard, Madeleine Gagnon, Denise Boucher, Louki Bersianik ou Yolande Villemaire prennent en charge l'inauthenticité inhérente au langage, superposant dans un "discontinuum» des réseaux de textes. Leurs paroles deviennent, et chacune dans sa voix, le centre opératoire de divers discours (ou types de discours) où le rire éclate comme un questionnement incessant du corps textuel collectif.

Le roman de Francine Noël participe de diverses façons à ce questionnement des textes culturels, y superposant, par un constant retour à l'histoire d'Elisa Doolittle, la problématique de la femme dans son accession au langage. Le paradigme du langage s'inscrit donc déjà dans une intertextualité «itinérante» ou réitérée par la traversée d'un même récit au cours des siècles. 
Ainsi, de l'antique Pygmalion à la pièce de Bernard Shaw et aux versions filmées d'Anthony Asquit et de Cukor (My fair lady) jusqu'à Maryse, se redit la montée d'une conscience par le biais de.l'amour et par l'apprentissage des langages de la culture dominante. Parcours d'une beauté (mythe grec) et d'une intelligence mise au monde par l'autre, jusqu'à son accession au contrôle parfait des valeurs du pouvoir et au débordement de ce contrôle dans la transgression.

Maryse, c'est justement cette pratique de la transgression impliquant la conscience d'une appartenance certaine aux cultures en circulation. Rupture qui ne peut se faire que dans/par le mouvement incessant où se jouent tous les textes. Maryse, c'est le rire du pastiche qui met en évidence et en perte les codes établis, mais c'est aussi le tragique d'une présence obsédante de ces textes-mythes-discours, facteurs d'une inscription culturelle souvent aliénante.

La citation, le pastiche et toutes ces réécritures formant «palimpseste» se présentent donc ici comme la prise en charge ou la trace d'une problématique du langage et de son rapport à l'aliénation. Tragique de femmes s'il en est.

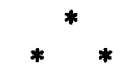

- Citez-moi donc Proust, Albertine disparue, édition Parpaillot, page quatre-vingt-dix-huit, sixième ligne, vite, rien que pour vérifier vos connaissances basiques.

- "La fille qui n'a point d'ami, répondit Maryse, Comment vit-elle? Elle ne dort jour ni demi Mais toujours veille. Ce fait amour qui la réveille Et qui la garde de dormir», Christine de Pisan, Encyclopédie Piperirvi, page trente-six.

Maryse, p. 64.

La citation avec bibliographie à l'appui est la part la plus explicite de l'intertextualité, sûrement aussi la plus conventionnelle. Ce qui se dégage pourtant de l'exergue précédent c'est que Maryse n'accepte de citer que dans la mesure où elle peut assumer le fragment emprunté, renvoyant à sa propre vie, ses insomnies, sa quête d'amour. Ce travail de la citation ${ }^{8}$ est donc un moyen de réactiver le sens des deux textes mis en présence et de les installer dans une complicité signifiante. Fonction première de la citation qui est de cautionner, par une similitude d'orientation ou d'idée, un fait textuel (ou fait d'existence pour les personnages).

Ce rôle élémentaire de l'intertextualité apparaît à quelques reprises dans le roman et permet, par $\wedge$ mouvement de déplacement qu'opère le contexte, de donner un nouvel éclairage aux fragments cités et d'introduire de part et d'autre un nouveau mode de lecture qui fait éclater la linéarité des textes.

Elle ouvrit son petit calepin et relut ce qu'elle y avait noté: "Les femmes et les prolétaires ne peuvent ni ne doivent devenir des auteurs pas plus qu'ils ne le veulent." C'était une citation que 
Simone de Beauvoir avait prise à Auguste Comte, et qui apparaissait à la page cent trente-six du Deuxième sexe. Depuis la veille, Auguste Comte était aux yeux de Maryse, le mal absolu.

Le texte qui sous-tend toute l'histoire de Maryse s'énonce lui aussi au discours direct dans les citations:

Soudain, une voix se mit à chanter:

"London Bridge is falling down,

falling down, falling down,

London Bridge is falling down,

My fair Lady".

Petite, elle avait longtemps pensé que la lady de la chanson était

Elisa Doolittle. A la fin de l'histoire de son père, le professeur

Higgins disait à Elisa: "Now Lisa, you are my fair lady..."

Le retour fréquent de la chanson l'installe dans le roman comme un leitmotiv. "Chanson thème", pourrait-on dire, qui renvoie constamment au processus d'identification entre Maryse et Elisa et, par là même, entre les deux textes qui les font exister. Cette référence centrale est d'ailleurs clairement approfondie et resituée en rapport avec de nombreux autres textes:

Comme si la femme devait être refaite par l'homme qui, de toute façon, lui donne toujours son statut social. Tel est le cas d'Elisa Doolittle, l'héroïne de Bernard Shaw, lequel soit dit en passant. n'a fait que broder sur le thème de Cendrillon. La Cendrillon de Perrault - ou celle de Straparole, c'est la même - réalise ses rêves de promotion sociale en étant "vue" par le Prince. "Peut-on être seulement vu? " dit Beckett.

Filiation intertextuelle qui, dans le parcours des citations prises comme supports idéologiques, pose l'appartenance des textes et des consciences à tout un tissu culturel. Cette présence soutenue d'une idéologie récurrente qui refoule le féminin hors des puissances productives prend une signification plus profonde dans la résurgence d'un même texte ou d'un même type de textes emprunté. Ainsi, par le truchement d'un choix d'éléments réalisé dans ce qu'on pourrait nommer le "texte public», Maryse retisse la trame d'une problématique féminine toujours réinvestie par les mots de l'Histoire, dénonçant, à travers l'insistance du retour, un étouffement symptomatique.

De même, le simple échantillonnage de titres ou de noms (auteurs/personnages) peut aussi renvoyer à tout un dire social et textuel. Des rapports se tissent de Macbeth (p. 294) à la Sagouine (p. 320) en passant par la sibylle de Cumes (p. 84) ou la Mère de Bertolt Brecht (p. 251). Coupable, sorcière, mère misérable, séductrice ou servante, une femme se dresse derrière chaque nom, une image surgit derrière chaque titre et parle sa multitude, greffant au texte la rumeur de toutes ces voix figées dans la répétition des figures symboliques, incessamment reprises par tous les modes de représentation. 
Si l'intertextualité se présente comme l'indice de la manière dont un texte lit l'histoire et s'écrit en elle, elle révèle aussi le pouvoir du texte comme formation et fixation des mentalités, matérialisant dans la citation ou la mention, cette constante référence au corps textuel. A ce réseau mobile dans lequel chaque texte renvoie aux autres en un dynamisme polyphonique s'ajoute la subtilité de l'allusion, trace d'une similitude et d'une connivence "discrète" entre deux discours.

Ainsi, les noms de Michel PARADIS, FRANÇOIS Ladouceur et de Télémaque SURPRENANT esquissent la silhouette éloquente de la belle Maria Chapdelaine, rivée à la terre et à sa condition. De même, Maryse, maigre et en attente, serveuse (provisoire) au Maplewood Inn, à l'enfance pauvre et au père oisif, projette l'ombre vibrante de Florentine Lacasse. Allusions qui s'inscrivent comme un souvenir, à l'instar de cette histoire toujours à réentendre d'Elisa Doolittle qui danse au bal et qui sait si bien parler de la pluie en Espagne (p. 138). Comme si, dans l'ombre, ces femmes de cultures et de conditions différentes redisaient une même affinité secrète, se liaient dans la reconnaissance d'un même lieu avant que jaillisse la voix. Comme si, entre le silence et la parole, entre l'inculture et l'université se jouait toujours la même scène: celle de l'amour et de l'appartenance. Nombreuses aussi sont les allusions à la "folie" des femmes (p. 56, 66, 141, 142, 177 ) et à ce "grand mystère" qui les entoure. Idées reçues trop souvent désolidarisées des conditionnements, des points de vue, et qui sont peut-être les plus grands textes de nos civilisations.

L'intertextualité devient donc aussi le rappel d'un éternel retour, le signe et la manifestation d'une répétition, d'une redite qui, adhésive ou subversive, ramène toujours la même question, celle d'une quête et de sa permanence. Mais il y a plus. Avec la conscience assumée des idéologies et des discours environnants, Maryse s'ouvre aussi à l'espace de la critique productive, à l'écriture pastichante qui attaque les textes de l'intérieur. Plaisir des «citations" parodiques qui mettent le discours dans une position critique vis-à-vis du modèle.

Le pastiche se présente comme une atteinte directe au langage dans sa manifestation stylistique/ formelle et, par là même, dans sa portée idéologique. Plus que tout autre procédé d'écriture, il révèle à quel point les jeux du signifiant, de la lettre, du mot, atteignent le signifié dans sa part la plus intime. Le texte modèle ou texte pastiché se fige en cliché et devient le corps et la matière de sa propre mythification. Ce travail du langage vient ici encore souligner, après les travaux de Derrida, Lacan et Serge Leclaire, que fondamentalement, rien n'échappe au mouvement du signifiant et que, en dernière instance, la différence entre le signifiant et le signifié n'est rien ${ }^{9}$.

Plus que tout autre dialogue avec le "texte public", le pastiche est, dans Maryse, le lieu d'une dénonciation des cultures et des contre-cultures qui leur appartiennent, le moment du rire qui devient le dire d'une anti-culture. Les discours s'excèdent dans la dérision et atteignent l'évidence de l'arbitraire et du figé propre à connoter toute spécificité culturelle. 
Déjà les titres des livres, seulement leurs titres la rebutaient et l'attiraient à la fois: ils lui semblaient, en quelque sorte, être permutables et se répondre les uns les autres (...). L'étrangeté de certains termes, qui revenaient comme en écho d'une couverture à l'autre, ne laissait pas de l'étonner. Elle en fit tout naturellement un pastiche intitulé Variations logogistiques.

Pastiche de titres. Délire du nom. Le titre comme nom du texte rattache celui-ci à tout un processus culturel, lui-même lié à un pouvoir informatif, persuasif, économique, donc profondément social. Par son style essentiellement elliptique qui ne peut que renvoyer au jeu et à l'illusion de la totalité, il semble que le titre se désigne comme le réceptacle "naturel» de l'idéologie, cette idéologie qui s'imprime davantage dans des représentations, des images ou des valeurs plutôt qu'en une philosophie.

Le titre est toujours en avant du texte. Pré-texte fétiche, il balance constamment entre un souhait d'ouverture et un effort de clôture. Pasticher les intitulés, c'est bien sûr révéler le pouvoir des textes ou des discours qu'ils évoquent, mais c'est surtout les surprendre dans leur tentative de possession du monde et dans leur besoin de s'ancrer dans un immobilisme «totalitaire». L'«intertitularité» dans la parodie s'attaque donc au lieu le plus conventionnel et le plus social de l'écriture. Sorte de "concentré» idéologique participant, par son constant retour, à l'emprise d'une épistémè, éclairant ainsi cet étrange écho d'une couverture à l'autre.

La signologie: science ou non-sens?

La transcendance du non-sens dans les sciences ancillaires. (...)

Transfert freudien, correspondance et ticket.

Calembours, jeux sur la double isotopie, allitérations, altérations, paronomases, etc., le déplacement qu'opère la magie des mots met en délire tous les discours didactiques, sémiotiques, structuraux, psychanalytiques, politiques, et fait apparaître cette étrange obsession et cet envahissement massif du langage technique spécialisé dans tous les domaines culturels. Le pastiche dans Maryse ne se limite d'ailleurs pas à ce petit exercice scolaire, mais parcourt le texte de long en large.

... département de "Français langue ornementale»

...étude sur le monoloy-québécois comme lieu d'embrayage privilégié des machines désirantes, orphelines et colonisées.

Le langage est joué, déjoué, dénoncé dans son arbitraire et son pouvoir dissimulé sous le masque de la spécificité et de l'hermétisme. Mais il n'y a pas que les discours intellectuels qui soient attaqués. Tout un système de dénomination sociale prend vie. Les prénoms, les noms de garderies (p. 240), de restaurants (p. 104), de parfums, de revues (p. 202) et autres «appareils» de la 
civilisation entrent aussi dans le tourbillon onomastique. Énumération extraordinairement prolifique trahissant cette manie obsédante du nom et de sa quête d'unicité. La nomination semble bien être, dans nos cultures, l'acte de langage par excellence assurant l'existence et la possession, et s'imposant souvent comme le seul rapport au vécu.

Derrière les titres et les noms se parlent aussi d'autres discours. En les parodiant, Maryse pose une fois de plus le roman comme reprise permanente mais déplacée des rhétoriques et des écritures. Les pastiches, dans leur profusion, empruntent à tous les textes. Mais les plus récurrents puisent dans ce qu'on pourrait appeler le langage du "nouvel intellectualisme", engendrant toute sa mythologie populaire.

De plus, la problématique de l'écriture est omniprésente dans Maryse, incarnée par son poète Oubedon et sa muse, référant ainsi, en la ridiculisant, à toute une aventure historique où l'homme artiste/écrivain trouve les ressources de son art dans la beauté suggestive d'une muse dévouée et fidèle. A cela s'ajoutent les discours psychanalytiques (p. 51), sémiotiques (p. 369), bureaucratiques, gynécologiques, les formulaires et fiches d'inscription et de renseignements (p. 240-242) et les ouvres de création: écrits de Maryse, poèmes de Oubedon, monologue de Marie-Lyre et le fameux récit de la Guerre des Murs, véritable épopée ubuesque (p. 205-223). Ce sont là autant de textes dans le texte se construisant sur le mode de la parodie.

Le paradigme du langage occupe donc une place centrale dans Maryse où il devient clair que s'approprier un pouvoir d'expression revient à s'approprier les valeurs qui s'y rattachent. À l'instar d'Elisa Doolittle, Maryse perçoit dans ce processus d'appropriation le moyen d'émerger d'une classe et d'une condition inférieure.

(Michel) aimait pourtant penser que sa blonde, née défavorisée, était maintenant de sa classe, et il avait raison: Maryse en reproduisait parfaitement les manières, le langage, le comportement, et elle faisait de son mieux pour en assimiler les valeurs.

Ce pouvoir d'imitation longtemps considéré comme "féminin" est profondément lié à une situation culturelle dans laquelle la femme n'avait, pour seul moyen d'accéder à une part d'existence, que celui d'imiter et de reproduire les discours du pouvoir. Accession qui devait toujours passer par l'autre. Elisa Doolittle et Maryse ne font que répéter cette attitude des dominés voués à penser leurs valeurs et à poser leurs gestes en fonction d'une dépossession initiale perpétrée par la classe dominante. Ce qui contribue sans doute à créer cet étrange paradoxe qui associe une certaine facilité pour les langues à des êtres qui ne pouvaient jamais la posséder. Problème qui se lit dans cette réflexion:

Étrangement, toutes les femmes de sa connaissance semblaient avoir un don pour les langues. Quel rapport entretenait-il luimême à cette langue dite maternelle et qui semblait être une histoire de femmes? "Serais-je un fifi?" se demanda-t-il soudain hagard. 
Cette situation associée à celle de tout un peuple longtemps considéré comme colonisé et se sous-estimant lui-même dans son rapport avec l'autre (c'est-à-dire tantôt le peuple anglais, mais surtout celui de France) et se raccrochant éperdument à sa culture, ne fait que redoubler le problème d'une incapacité de s'assumer dans sa différence.

On prenait des pots à l'époque: Lemire était français et tout le monde de la gang parlait comme lui.

Pour Maryse comme pour Elisa, l'acquisition du langage est débordée par une volonté et une capacité soudaine d'indépendance. Mais pour la première, la transgression finale est contemporaine d'une pratique de l'écriture cherchant sa part entière pour qu'enfin elle ait lieu.

Écrire, nous l'avons dit, c'est réécrire. Mais il y a, par-delà la répétition, un moment, une "chance" de déconstruire les valeurs et les discours acquis. Maryse manifeste ainsi, par son travail intertextuel et parodique, la dénonciation et le débordement des instances dominantes, la conquête infinie d'un lieu, DU lieu de rupture, c'est-à-dire celui, distanciateur, du jeu.

De la référence citationnelle ou allusive au pastiche de titres et à celui des discours, Maryse opère la traversée des langages et leurs retournements. Le pastiche ne pouvant venir qu'après la possession, il devient le signe d'une libération. Dans le roman il atteint un sommet lorsque Maryse se l'approprie pour annoncer son choix d'indépendance:

Je suis le foyer de cristallisation de la mauvaise conscience petitebourgeoise. Tu comprends, minou, chez-moi, l'instance représentative a toujours été doublée d'entrée de jeu, si je puis dire - et ça je m'en doute depuis la fin de ma cure au monoglutamate de sodium -, cette instance, dis-je, a toujours été doublée d'une instance auto-réfringante profondément castratrice... etc.

Que dire sinon qu'il s'agit là d'une aventure du langage qui, de l'imitation à l'assimilation, accède enfin à la jouissance et à l'excès. Les jeux de mots, les distorsions morphologiques et syntaxiques deviennent une véritable exploration critique et ludique. "Trip de langage", Maryse est l'écriture de la jouissance pour la mise au monde d'un texte qui met en état de perte (...), qui déconforte (...) et fait vaciller les assises historiques, culturelles, psychologiques du lecteur (...), met en crise son rapport au langage ${ }^{10}$.

Et, comme toute jouissance, cette écriture s'articule sur le fond tragique de la mort. Mort dont nous n'avons pas parlé mais qui est sans doute la part du monde et du texte la plus envahissante puisqu'elle est toujours le non-dit et la raison d'être de tout discours. Elle transparaît en tout premier lieu, dans l'exergue qui devance et oriente le roman: Mais la mort, pour moi, ce n'est pas quelque chose dont on peut parler (Marie Uguay). 
Le carnavalesque qui ressurgit à chaque renversement des codes et discours devient donc peut-être le dernier recours dans un univers où une parole toujours déjà dite impose la dissolution d'un ego souverain et maître du sens. Le pastiche, la mise en déroute des stéréotypes, l'imitation parodique deviennent les matériaux de la distanciation d'un sujet qui cherche peut-être désespérément à s'affirmer.

On se souvient de Bérénice Einberg et du «bérénicien». Le tragique de Maryse, s'il n'a pas la violence sauvage de l'avalée des avalées, se situe lui aussi dans un ludisme qui ne cherche pas à stopper l'avalement mais à le multiplier et à le dévier pour en révéler le pathos. Maryse, c'est la "grande bouffe" des discours envahissants, se saisissant des codes, les transformant dans une profusion délirante qui, elle, devient la nouvelle affirmation d'une unicité. Nouveau sujet qui s'instaure à partir de sa propre déconstruction.

Plusieurs écritures contemporaines travaillent aujourd'hui dans cette direction, indiquant peut-être par là une sorte de récupération épistémique. Inévitable? Il reste que le roman québécois de ces dernières années accepte, comme Maryse, de parler à partir d'un lieu éclaté par des voies de moins en moins territoriales. Il s'inscrit dans cette simultanéité des voix qui refuse l'immobilisme du sens.

Maryse et Bérénice: peut-être la part brimée de la parole rejaillissant pour faire sens dans une circulation permanente/permutante des déjà-dits. La part du fou.

1. Francine Noël, Maryse, Montréal, VLB éditeur, 1983, 426 p.

2. Mikhail Bakhtine, Problèmes de la poétique de Dostoïevski, Lausanne, L'Âge d'Homme, 1970, $317 \mathrm{p}$.

3. Julia Kristeva, la Révolution du langage poétique, Paris, Seuil, 1974.

4. Id., Semeiotikè, Paris, Seuil, 1978.

5. Linda Hutcheon, A Rhetoric of Irony, Chicago et Londres, University of Chicago Press, 1974, et «Ironie et parodie: stratégie et structure» in Poétique no 36, novembre 1978, p. 467-477.

6. Ibidem, p. 470.

7. Evelyn Voldeng, "L'Intertextualité dans les écrits féminins d'inspiration féministe» in Voix et images, vol. VII, no 3, printemps 1982.

8. Antoine Compagnon, la Seconde main ou le travail de la citation, Paris, Seuil, 1979: La citation travaille le texte, le texte travaille la citation. Ici surgit le sens... p. 36.

9. Jacques Derrida, De la grammatologie, Paris, Minuit, 1967, p. 36.

10. Roland Barthes, le Plaisir du texte, Paris, Seuil, 1976, p. 25. 